\title{
Research
}

\section{Diagnostic approach to urinary tract infections in male general practice patients:}

\author{
a national surveillance study
}

\begin{abstract}
Background

Diagnostic urinary tract infection (UTI) studies

have primarily been performed among female patients.
\end{abstract}

\section{Aim}

To create a diagnostic algorithm for male general practice patients suspected of UTI.

\section{Design and setting}

Surveillance study in the Dutch Sentinel General Practice Network

\section{Method}

Clinical information and dipstick results were collected from 603 patients. Algorithmpredicted care was compared with care as usual in terms of sensitivity lantibiotic recommended when UTI was confirmed) and specificity (no antibiotic recommended when no UTI was observed].

\section{Results}

Complete information was available from $490 / 603$ (81\%) males, of whom $66 \%$ (321/490) had a UTI. A diagnostic algorithm recommending antimicrobial prescription in the case of a positive nitrite test or a positive leukocyte esterase test in males aged $\geq 60$ years, had a positive predictive value (PPV) of $83 \%[95 \%$ confidence interval $[\mathrm{Cl}]=78$ to 87 and a negative predictive value (NPV) of $60 \%$ $(95 \% \mathrm{Cl}=52$ to 66$)$, respectively larea under the ROC curve: $0.78,95 \% \mathrm{Cl}=0.74$ to 0.82 ). When both dipstick results were positive in males aged $\geq 60$ years, PPV increased to $90 \% 195 \% \mathrm{Cl}$ $=83$ to 94), whereas NPV was highest in males $<60$ years with negative dipstick results $171 \%$, $95 \% \mathrm{Cl}=59$ to 81 ). Sensitivity and specificity of predicted UTI care and usual care did not differ $175 \%$ versus $79 \%, P=0.30$, and $70 \%$ versus $63 \%, P=0.17$, respectively)

\section{Conclusion}

UTI care provided to Dutch male GP patients is as accurate as predicted care from a diagnostic algorithm. The studied clinical information and dipstick tests are useful for ruling in UTI in males, but have limited value in ruling out this diagnosis.

\section{Keywords}

general practice; male; patients; urinary tract infections.

\section{INTRODUCTION}

Male urinary tract infections (UTI) are not as frequently observed as female UTIs, although with increasing age this bacterial infection also becomes more common in males. In The Netherlands, the average annual incidence rate of male UTIs was 156 per 10000 compared with 922 per 10000 females in 2010.' At the age of 85 , the incidence rate in males was 1319 per 10000 , thus exceeding the average annual incidence rate of female UTIs.

In general practice patients suspected of having a UTI, the evaluation of the predictive value of diagnostic information (such as clinical symptoms of UTI and dipstick results) has been performed primarily for females. ${ }^{2,3}$ The rationale of these studies is to provide clinicians with a clinical decision rule to improve the quality of UTI care.4,5 Mclsaac etalhave shown that the application of a simple diagnostic algorithm can result in the correct treatment of most females with UTI and can reduce unnecessary urine culture testing and antibiotic prescriptions. ${ }^{5}$

Comparable studies of UTI predictors have seldom been performed for male general practice patients. As a consequence, guidelines for diagnosis and treatment of UTI in males are mainly based on female studies, although one can question whether the generalisation of these criteria to males is valid due to the clear genito-urinary differences. ${ }^{6}$ A few diagnostic male UTI studies have addressed the predictive value

CDJ den Heijer; MD, PhD student

EE Stobberingh, PhD, medical microbiologist Maastricht University Medical Centre/CAPHR Maastricht, the Netherlands. MCJM van Dongen, MD, PhD, clinical epidemiologist, Faculty of Health, Medicine and Life Sciences, Maastricht University,

Maastricht, The Netherlands. GA Donker. MD PhD, GP, coordinator of Sentinel General Practice Network, the Netherlands Institute for Health

Services Research, Utrecht, the Netherlands.

Address for correspondence

Ellen E Stobberingh, Maastricht University of dipstick test results, but conclusions were contradictory. ${ }^{6,7}$ Assessment of clinical symptoms was considered in only one of these studies, based on a relatively small sample size $(n=90)$.?

In addition to the development of a diagnostic algorithm, the potential gain in the quality of UTI care to be attributed to the use of the newly developed rule needs to be assessed in clinical practice. ${ }^{5}$

For these reasons, the study developed a diagnostic UTI algorithm using clinical information and dipstick results from Dutch male general practice patients with symptoms indicative of UTI. The study also assessed the potential impact of the newly developed diagnostic UTI algorithm by comparing its medical decisions, that is, prescription of antimicrobial therapy, with usual care.

\section{METHOD}

Patients

Dutch general practices ( $n=42)$ participating in the NIVEL Sentinel General Practice Network were used for patient recruitment. This network accounts for $~ 0.8 \%$ of the Dutch population and is nationally representative by age, sex, regional distribution and population density.

From January 2009 to June 2011, GPs included male general practice patients ( $\geq 18$ years) with symptoms of UTI (dysuria, urinary frequency and/or urgencyl. Patients having urological or nephrological
Medical Centre, Department of Medical Microbiology, PO Box 5800, 6202AZ Maastricht, The Netherlands.

E-mail: e.stobberinghamumc.nl

Submitted: 15 March 2012; Editor's response: 12 April 2012; final acceptance: 15 May 2012 @British Journal of General Practice

This is the full-length article lpublished online 29 Oct 2012) of an abridged version published in print. Cite this article as: Br J Gen Pract 2012; DOI: 10.3399/bjgp12X658313 


\section{How this fits in}

Studies on the evaluation of diagnostic information including patients suspected of a urinary tract infection (UTI) aim to improve the quality of UTI care. Adequately powered studies of UTI predictors have not been performed for male general practice patients. The present study shows that using clinical information and dipstick tests, ruling in a UTI in males can be achieved. Actual care provided by Dutch GPs to males suspected of UTI was as accurate as recommended care based on a diagnostic algorithm.

comorbidities (with the exception of benign prostate hypertrophyl, diabetes mellitus or other immunocompromising diseases were excluded, as well as those catheterised or suspected of having a sexually transmitted disease.

\section{Clinical information and dipsticks}

For each eligible patient, the GP filled in a questionnaire giving clinical patient information, that is, age, history of UTI in the past year, and the presence or absence of dysuria, urinary frequency and/or urgency, fever $\left(>38^{\circ} \mathrm{C}\right)$, and flank pain. These clinical symptoms were derived from the Urinary Tract Infection Guidelines compiled by the Dutch College of General Practitioners (NHG). ${ }^{8}$

All patients provided a fresh-voided midstream urine sample as part of routine clinical practice. The GP or practice assistant used this sample to perform a dipstick test for nitrite and leukocyte esterase (LE) activity. Any change in colour was considered to be a positive test result. ${ }^{9}$ Finally, GPs recorded the empirical antimicrobial treatment prescribed. The GPs choice to prescribe an antibiotic lyes/ no) empirically was considered to be the 'care as usual'.

\section{Urine culture}

From the obtained urine sample, a dipslide Uriline, 56508, Biomérieux, Plainview, NY, US) was inoculated for all included males according to the manufacturer's instructions and sent to the Department of Medical Microbiology, Maastricht University Medical Centre, The Netherlands. The gold standard to diagnose a microbiologicallyconfirmed UTI in males is uropathogen growth of $\geq 103$ colony-forming units per $\mathrm{ml}$ (cfu/ml). ${ }^{10}$ Standard microbiological methods were used for the identification of uropathogens. ${ }^{11}$

\section{Predictors of UTI}

Diagnostic algorithms were developed for clinical information and dipstick results separately and combined. Model development was performed according to the procedure described by Little et al, with minor modifications. ${ }^{12}$ In short, to identify relevant predictors of UTI, multivariable logistic regression analyses were carried out from which only the statistically significant predictors $(P<0.05)$ were included in the final prediction models. In these analyses, patient's age was dichotomised using the most discriminative cut-off value $(60$ years of age). Weights were given to all significant predictors based on the obtained beta coefficients and these weights were used to assign 'weight scores' to all individual patients.

To assess the best performing algorithm, the following parameters were determined for each model, using all possible weight scores as cut-off points: sensitivity, specificity, positive and negative predictive values (PPV and NPV respectively), likelihood ratios for a positive (LR+) and a negative (LR-) test result. Also, the proportion of patients at or above the cutoff value was assessed, which corresponds to the proportion of patients for whom the algorithm would have recommended an empirical antimicrobial agent. Predictive values were most discriminative in the assessment of the best performing algorithm. ROC curves of sensitivity versus 1 - specificity were plotted using a webbased calculator. ${ }^{13}$

\section{Potential impact of diagnostic algorithm:} comparison of predicted care with usual care

The potential impact of the diagnostic algorithm was determined by comparing the sensitivity and specificity of the algorithmdriven prescription strategy with care as usual. $^{5}$

Sensitivity was defined as the proportion of males with a microbiologically-confirmed UTI for whom an antimicrobial prescription was recommended. Specificity was defined as the proportion of males without a microbiologically-confirmed UTI for whom an antimicrobial prescription was not recommended.

A $P$-value $<0.05$ was considered statistically significant. The programme SPSS (16.0 for Windows) was used for statistical analyses.

\section{RESULTS}

\section{Patients}

In total, 603 male patients participated with a 
Table 1. Clinical weight scores ${ }^{\mathrm{a}}$ to predict a microbiologically-confirmed UTI

\begin{tabular}{|c|c|c|c|c|c|c|c|}
\hline $\begin{array}{l}\text { Cut-off point ( } \% \text { at or } \\
\text { above cut-off point) }\end{array}$ & $\begin{array}{l}\text { Sensitivity } \\
\text { (95\% CI] }\end{array}$ & $\begin{array}{l}\text { Specificity } \\
(95 \% \mathrm{CI})\end{array}$ & $\begin{array}{c}\text { PPV } \\
(95 \% \mathrm{Cl})\end{array}$ & $\begin{array}{c}\text { NPV } \\
(95 \% \mathrm{Cl})\end{array}$ & $\begin{array}{c}\text { Correctly } \\
\text { classified \% }\end{array}$ & $\begin{array}{c}\text { LR+ } \\
(95 \% \mathrm{Cl})\end{array}$ & $\begin{array}{l}\text { LR- } \\
(95 \% \mathrm{Cl})\end{array}$ \\
\hline$\geq 0(100 \%)$ & 100 & 0 & - & - & 65.5 & 1 & - \\
\hline$\geq 0.5$ (86.7\%), D & 91 (88 to 94) & 22 (16 to 29) & 69 (64 to 73 ) & 57 (44 to 69) & 67.3 & 1.17 (1.07 to 1.27$)$ & 0.40 (0.27 to 0.59$)$ \\
\hline$\geq 1(66.3 \%), F / A$ & 75 (70 to 79 ) & 50 (42 to 58) & 74 (69 to 79 ) & 51 (43 to 59) & 66.1 & 1.49 (1.26 to 1.75$)$ & 0.51 (0.42 to 0.62 ) \\
\hline$\geq 1.5(39.8 \%), F / A+D$ & 48 (42 to 53) & 75 (68 to 81 ) & 79 (72 to 84 ) & 43 (37 to 49) & 57.1 & 1.92 (1.44 to 2.55$)$ & 0.70 (0.63 to 0.78$)$ \\
\hline$\geq 2(8.2 \%), F+A$ & 11 (8 to 15 ) & 96 (92 to 99) & 85 (70 to 94 ) & 36 (32 to 41$)$ & 40.2 & 2.98 (1.28 to 6.96$)$ & 0.93 (0.89 to 0.96$)$ \\
\hline$\geq 2.5(4.9 \%), F+A+D$ & 7 (4 to 10$)$ & 99 (95 to 100) & 92 (72 to 99) & 36 (32 to 40 ) & 38.6 & 5.79 (1.38 to 24.3 ) & 0.94 (0.91 to 0.97) \\
\hline
\end{tabular}

${ }^{a}$ Derived from sum of rounded logistic coefficients: dysuria 0.5, fever 1.0, and age $>60$ years 1.0. ${ }^{b}$ Based on a cut-off value of $\geq 103$ colony-forming units per ml. $A=$ age $>60$ years. $D=$ dysuria. $F=$ fever $. \angle R+=$ likelihood ratio for a positive test. $L R-=$ likelihood ratio for a negative test. NPV $=$ negative predictive value. PPV $=$ positive predictive value. UTI = urinary tract infection.

median age of 65 years (range 18-97 years). Clinical information was recorded from 562 of 603 males (93\%) and dipstick results were obtained from $87 \%$ (527/603) of all included males.

Only patients with complete data on clinical information and dipstick results (81\%, 490/603) were considered eligible for analysis, of whom 66\% (321/490) had a microbiologically-confirmed UTI. GPS prescribed an antimicrobial agent to 315 of these 490 male patients (64\%).

\section{Influence of missing data}

The age of patients with missing data was comparable to the analysed population: median age of 66 years (range 18-90 years). To assess the influence of missing data, all performed analyses were also done on the sample with either complete clinical ( $n=562)$ or dipstick ( $n=527)$ information. In comparison with the presented results, dysuria lodds ratio [OR] $1.41,95 \% \mathrm{Cl}=$ 0.97 to 2.04) was not predictive for UTI when analyses included all 562 patients with clinical information. For the other predictors, the results did not differ substantially between the sample with complete clinical and dipstick information and those with only complete clinical or dipstick information (data not shown).

\section{Clinical information predictive for UTI}

Dysuria (OR 1.57, 95\% Cl=1.05 to 2.36; weight score: 0.5 ), fever (OR $2.46,95 \% \mathrm{Cl}=1.22$ to 4.96; weight score: 1.0 ) and age $\geq 60$ years IOR $2.49,95 \% \mathrm{Cl}=1.66$ to 3.74 ; weight score: 1.0) were found to be predictors for UTI when considering clinical information alone. A weight score of one was the best cut-off value for the prediction model with PPV and NPV of $74 \%$ and $51 \%$, respectively (Table 1). This model corresponds to an algorithm recommending antimicrobial prescriptions for males aged $\geq 60$ years or those with fever. When all three clinical characteristics were present, the predictive value for having UTI was $92 \%$. But patients with none of these three clinical characteristics still had a chance of $43 \%$ of having UTI.

\section{Dipstick results predictive for UTI}

UTI was associated with positive nitrite $10 \mathrm{R}$ $8.18,95 \% \mathrm{Cl}=4.77$ to 14.03 ; weight score: 2.0) and LE tests (OR $1.80,95 \% \mathrm{Cl}=1.16$ to 2.80; weight score: 1.0$)$. When the algorithm was based on a weight score of two Irecommending prescriptions to patients with a positive nitrite test), PPV was $90 \%$ and NPV 52\% (Table 2). In 91\% of the cases with both nitrite and LE test results positive, UTI was confirmed. With both tests negative, $40 \%$ still had a positive urine culture.

\section{Table 2. Dipstick weight scores ${ }^{a}$ to predict a microbiologically-confirmed UTI ${ }^{b}$}

\begin{tabular}{|c|c|c|c|c|c|c|c|}
\hline $\begin{array}{l}\text { Cut-off point (\% at or } \\
\text { above cut-off point) }\end{array}$ & $\begin{array}{l}\text { Sensitivity } \\
\text { (95\% CI) }\end{array}$ & $\begin{array}{l}\text { Specificity } \\
(95 \% \text { CI) }\end{array}$ & $\begin{array}{l}\text { PPV } \\
(95 \% \mathrm{Cl})\end{array}$ & $\begin{array}{c}\text { NPV } \\
(95 \% \mathrm{Cl})\end{array}$ & $\begin{array}{l}\text { Correctly } \\
\text { classified } \%\end{array}$ & $\begin{array}{l}\text { LR+ } \\
(95 \% \mathrm{Cl})\end{array}$ & $\begin{array}{l}\text { LR- } \\
(95 \% \text { CI) }\end{array}$ \\
\hline$\geq 0(100 \%)$ & 100 & 0 & - & - & 65.5 & 1 & - \\
\hline$\geq 1$ (71.0\%), L & 82 (78 to 86 ) & 50 (43 to 58) & 76 (71 to 80 ) & 60 (51 to 68$)$ & 71.2 & 1.65 (1.41 to 1.94$)$ & 0.35 (0.28 to 0.45 ) \\
\hline$\geq 2$ (66.3\%), N & 58 (52 to 63) & 88 (82 to 92) & 90 (85 to 94) & 52 (46 to 58 ) & 68.2 & 4.87 (3.19 to 7.43 ) & 0.48 (0.42 to 0.55 ) \\
\hline$\geq 3$ (37.3\%), L+N & 52 (46 to 57 ) & 90 (84 to 94) & 91 (85 to 94) & 50 (44 to 55$)$ & 64.9 & 5.14 (3.24 to 8.17) & 0.54 (0.48 to 0.60 ) \\
\hline
\end{tabular}

${ }^{a}$ Derived from sum of rounded logistic coefficients: leucocyte 1.0 and nitrite 2.0. ${ }^{b}$ Based on a cut-off value of $\geq 103$ colony-forming units per $m l . L=l e u c o c y t e . ~ L R+=l$ likelihood ratio for a positive test. $L R-=$ likelihood ratio for a negative test. $N=$ nitrite. $N P V=$ negative predictive value. $P P V=$ positive predictive value. $U T I=$ urinary tract infection. 
Table 3. Clinical symptoms and dipstick results combined, predictive for UTla

\begin{tabular}{|c|c|c|c|c|c|}
\hline $\begin{array}{l}\text { Diagnostic information } \\
\left.\text { (coefficient }{ }^{b}\right)\end{array}$ & $\begin{array}{c}\mathrm{UTI}^{\mathrm{a}}(n=321) \\
n(\%)\end{array}$ & $\begin{array}{c}\text { No UTI }(n=169) \\
n(\%)\end{array}$ & $\begin{array}{l}\text { Unadjusted OR } \\
\text { (95\% CI) }\end{array}$ & $\begin{array}{l}\text { Adjusted OR } \\
(95 \% \mathrm{CI})\end{array}$ & $\begin{array}{c}\text { Weights }{ }^{c} \text { in } \\
\text { prediction model }\end{array}$ \\
\hline Urinary frequency & $129(40)$ & 57 (34) & 1.32 (0.90 to 1.95$)$ & 0.83 (0.51 to 1.34 ) & - \\
\hline Dysuria & $194(60)$ & $85(50)$ & $1.51(1.04$ to 2.20$)$ & 1.35 (0.86 to 2.11$)$ & - \\
\hline Urinary urgency & 107 (33) & $38(22)$ & 1.72 (1.12 to 2.65 ) & 1.40 (0.84 to 2.33 ) & - \\
\hline Fever & $50(16)$ & $11(7)$ & 2.65 (1.34 to 5.24$)$ & 1.58 (0.73 to 3.39 ) & - \\
\hline Flank pain & $47(15)$ & 26 (15) & 0.94 (0.56 to 1.59 ) & 1.42 (0.77 to 2.63 ) & - \\
\hline Age $>60$ years $(0.75)$ & $224(70)$ & $80(47)$ & 2.57 (1.75 to 3.77$)$ & $2.11(1.35$ to 3.31$)$ & 1 \\
\hline UTI history in previous year & 103 (32) & $38(22)$ & $1.63(1.06$ to 2.51$)$ & 1.03 (0.62 to 1.71$)$ & - \\
\hline Nitrite (2.02) & $185(58)$ & 20 (12) & 10.13 (6.05 to 16.99 ) & 7.54 (4.34 to 13.09) & 2 \\
\hline Leucocyte (0.52) & $245(76)$ & $81(48)$ & $3.50(2.36$ to 5.21$)$ & $1.68(1.06$ to 2.69$)$ & 1 \\
\hline
\end{tabular}

${ }^{a}$ Based on a cut-off value of $\geq 103$ colony-forming units per $\mathrm{ml} .{ }^{b}$ Coefficients are only given for significant predictors. ${ }^{c}$ Weights are based on the coefficients from multivariable regression analysis. OR = odds ratio. UTI = urinary tract infection.

\section{Table 4. Combined dipstick and clinical weight scores ${ }^{a}$ to predict a microbiologically-confirmed UTI ${ }^{b}$}

\begin{tabular}{|c|c|c|c|c|c|c|c|}
\hline $\begin{array}{l}\text { Cut-off point ( } \% \text { at } \\
\text { or above cut-off point) }\end{array}$ & $\begin{array}{l}\text { Sensitivity } \\
(95 \% \mathrm{Cl})\end{array}$ & $\begin{array}{l}\text { Specificity } \\
(95 \% \mathrm{CI})\end{array}$ & $\begin{array}{c}\text { PPV } \\
(95 \% \text { CI) }\end{array}$ & $\begin{array}{c}\text { NPV } \\
(95 \% \text { CI) }\end{array}$ & $\begin{array}{l}\text { Correctly } \\
\text { classified \% }\end{array}$ & $\begin{array}{c}\text { LR+ } \\
(95 \% \mathrm{Cl})\end{array}$ & $\begin{array}{l}\text { LR- } \\
(95 \% \text { CI) }\end{array}$ \\
\hline$\geq 0$ (100\%) & 100 & 0 & - & - & 65.5 & 1 & - \\
\hline$\geq 1$ (85.3\%), L/A & 94 (90 to 96 ) & 30 (24 to 38 ) & 72 (67 to 76 ) & 71 (59 to 81) & 71.2 & 1.34 (1.21 to 1.48$)$ & 0.22 (0.14 to 0.34$)$ \\
\hline$\geq 2(59.6 \%), N$ or $L+A$ & 75 (70 to 80 ) & 70 (62 to 77 ) & 83 (78 to 87) & 60 (52 to 66 ) & 73.3 & 2.49 (1.96 to 3.16$)$ & 0.36 (0.29 to 0.43 ) \\
\hline$\geq 3(40.4 \%), N+L / A$ & 56 (50 to 61) & 89 (83 to 93) & 90 (85 to 94 ) & 51 (46 to 57) & 67.1 & 4.96 (3.21 to 7.66 ) & 0.50 (0.44 to 0.56$)$ \\
\hline$\geq 4(26.9 \%), N+L+A$ & 37 (32 to 43) & 92 (87 to 96) & 90 (83 to 94) & 44 (38 to 49) & 56.1 & 4.82 (2.80 to 8.28 ) & 0.68 (0.63 to 0.74$)$ \\
\hline
\end{tabular}

${ }^{a}$ Derived from sum of rounded logistic coefficients: leucocyte 1.0, age $>601.0$ and nitrite $2.0 .{ }^{b}$ Based on a cut-off value of $\geq 103$ colony-forming units per $\mathrm{ml}$. $A=$ age $>60$ years. $L=$ leucocyte. $L R+=$ likelihood ratio for a positive test. $L R-=$ likelihood ratio for a negative test. $N=$ nitrite. $N P V=$ negative predictive value. $P P V=$ positive predictive value. UTI = urinary tract infection.

\section{Clinical information and dipstick results combined}

Age $\geq 60$ years and positive dipstick results were still predictive for UTI when all diagnostic information was combined in one model (Table 3). When antimicrobial prescription was recommended in the case of a positive nitrite test or a positive LE test in males aged $\geq 60$ years (representing a weight score of 2), the PPV and NPV were $83 \%$ and $60 \%$ respectively (Table 4 ). If both dipstick results were positive in males aged $\geq 60$ years, UTI was microbiologically confirmed in $90 \%$ of the cases. UTI was observed in $29 \%$ of males $<60$ years with negative dipstick results.

Potential impact of diagnostic algorithm: predicted care compared with usual care ROC curve analysis revealed the highest accuracy for the combined model (Figure 1) and this was thus regarded as the best model. The derived algorithm is presented in Figure 2. No difference was observed between the proportions of males with UTI for whom antimicrobial prescription was recommended by this algorithm and the care as usual (75\% versus $79 \%$ respectively, $\chi^{2}=1.06, P=0.30$ ). The same applied to the proportions of males without UTI for whom antimicrobial prescription was not recommended $170 \%$ versus $63 \%$ respectively, $\chi^{2}=1.91, P=0.17$ ).

\section{DISCUSSION}

\section{Summary}

In male general practice patients suspected of UTI, actual care provided by Dutch GPs was as accurate as recommended care based on a diagnostic algorithm. This was assessed by comparing the sensitivities and specificities of both strategies, with the preferred prescription policy based on culture results as the gold standard. Using clinical information and dipstick results, the presence of male UTIs can be predicted correctly.

\section{Strengths and limitations}

To the best of the authors' knowledge, this is the first adequately powered study that addressed both dipstick results and 


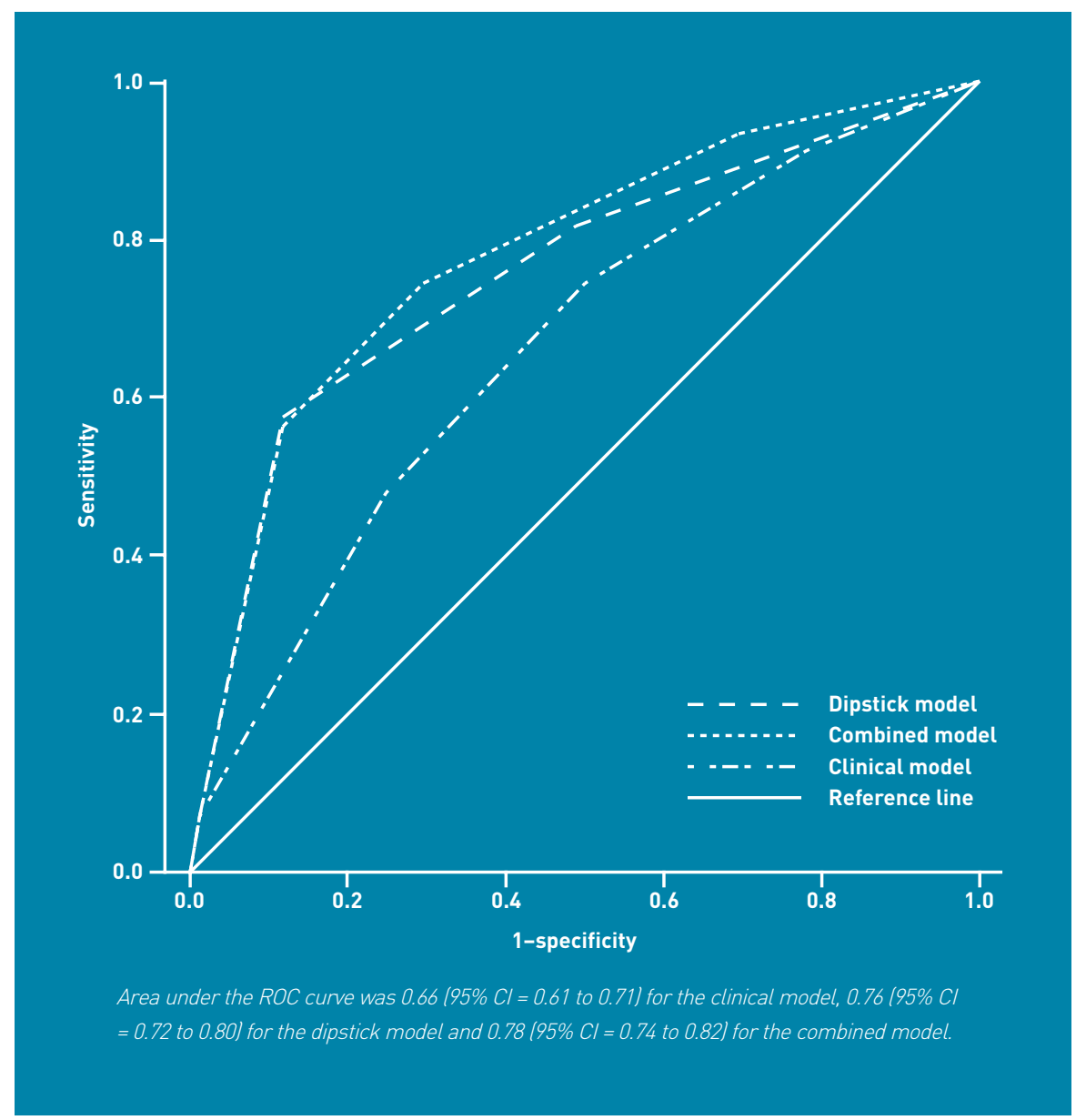

Figure 1. ROC curve analyses of the three UTI prediction models based on clinical symptoms, dipstick results and both together.

Figure 2. Diagnostic algorithm for male general practice patients suspected of UTI. UTI = urinary tract infection. algorithm for UTI in male general practice patients. In addition, the use of a Sentinel General Practice Network, which covers a nationally representative patient population, makes the results applicable to the whole Dutch general practice population.

It has been reported that the severity of clinical information in making a diagnostic

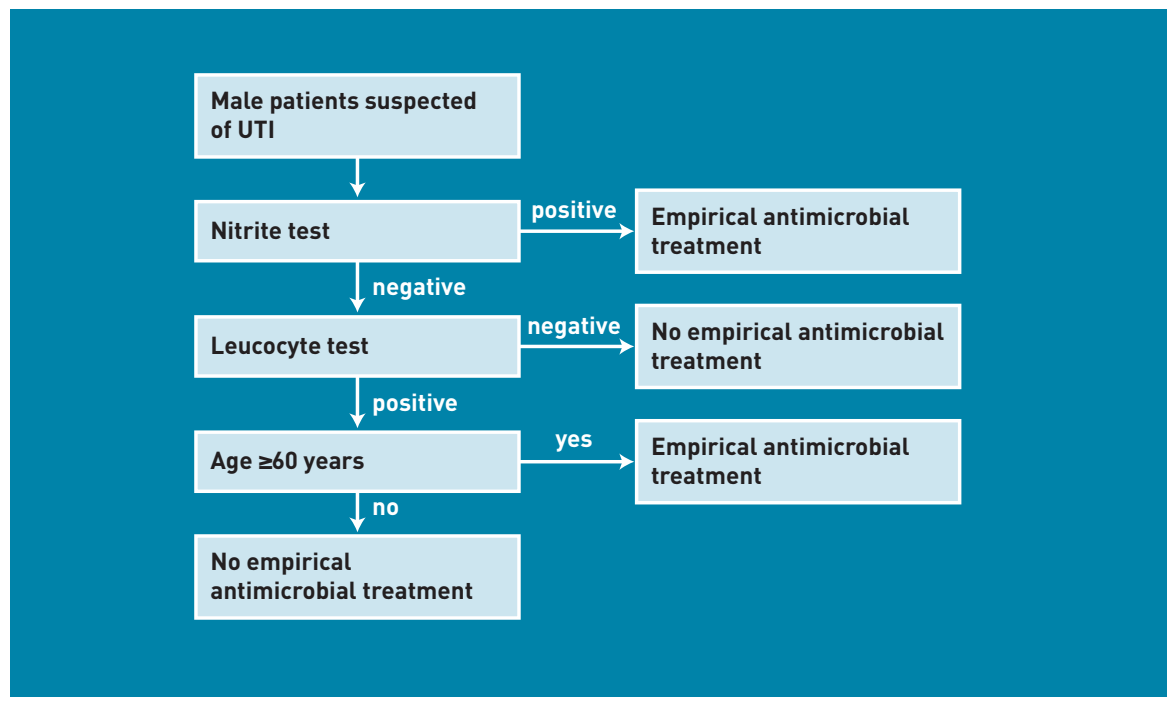

symptoms is more predictive for UTI than the actual presence of symptoms. ${ }^{14}$ Since no validated questionnaires were available to assess the severity of male UTI symptoms, it was decided not to include it in the design. In addition, the recorded diagnostic information was in accordance with the Dutch Urinary Tract Infection guidelines and therefore other information that could have predictive value, like haematuria, was not included in the analyses. ${ }^{8,14}$ Although 19\% of the data was missing, consequences for the current analyses were limited. Only the predictive value of dysuria for the presence of UTI should be interpreted with caution, as this symptom was not discriminative for UTI when all patients with clinical information available were considered, irrespective of the presence of dipstick data.

\section{Comparison with existing literature}

For females suspected of UTI, clinical decisions based on a diagnostic algorithm have been compared with care as usual by Mclsaac et al. ${ }^{15}$ In their algorithm, antimicrobial prescription was recommended when at least two of the following clinical findings were present in females suspected of having a UTI: symptoms for 1 day, dysuria, positive leukocyte or nitrite test. The number of prescriptions in the case of a negative culture result could be reduced by $40 \%$ if their algorithm was implemented. However, this benefit was mainly attributable to the high antimicrobial prescription rate of the participating GPs (89\%) as the performance of their algorithm was similar to this study in terms of sensitivity and specificity ( $80 \%$ versus $75 \%$ and $54 \%$ versus $70 \%$, respectively).

Compared with the results, HummersPradier et al have reported considerably lower positive predictive values for nitrite (90\% versus $78 \%$ ) and LE (76\% versus $65 \%$ ) tests in males.? Although UTI prevalence was similar in both settings $165 \%$ versus $60 \%)$, their patient population included males with comorbidity, for example, catheterised patients and males with diabetes mellitus, which could account for the observed differences. Also, their small sample size $(n=90)$ yielded parameters with large Cls. In a 2004 study, using the same network as in the present study and including 422 males, reported PPVs for nitrite $(96 \%)$ and LE $(71 \%)$ were similar to this present study. ${ }^{6}$

The value of age in predicting UTI in males was earlier reported by Hummers-Pradier, who also observed a positive association between age $\geq 60$ years and UTI. ${ }^{7}$ Although 
this study is aware that a positive urine culture in older people must be interpreted with caution because of the relatively high rate of contamination, this is mainly true for impaired older people and those who are living in long-term care facilities. ${ }^{16}$ In this study population, a relatively healthy, homogeneous patient population was included because of the in- and exclusion criteria used, decreasing the likelihood of contamination.

\section{Implications for research and practice}

'Ruling in' UTI diagnosis in males can be done by using dipstick test results, given the high probability of UTI when results, and more specifically the nitrite test, are positive. This supports the previously stated recommendation to start empirical treatment in males suspected of UTI with a positive nitrite test. ${ }^{6}$ When dipstick results are unknown, GPs can be quite confident in males $\geq 60$ years with dysuria and fever, that a UTI will later be confirmed microbiologically, justifying empirical treatment.

However, 'ruling out' UTI was more problematic, due to the relatively high probability of UTI even when all predictors were absent. Negative dipstick results combined with a relatively low age (<60 years) reduce the probability of a UTI compared with negative dipstick results alone (29\% versus $40 \%$ ). However, this chance was considered to be too high still to rule out UTI and therefore opt for urine culture in these patients.

Nonetheless, the study does acknowledge that the study protocol, with the inclusion of variables predominantly aimed to rule in UTI, will have influenced results in favour of ruling in a UTI and will, consequently, have less power to rule out this diagnosis.

Therefore, more discriminative diagnostic information for male UTIs is needed and, in particular, information that can rule out UTI. In this perspective, a questionnaire that is sensitive to changes in the symptomatology of UTI in males needs to be developed and tested psychometrically. Also, clinical prediction models need external validation, as the original sample tends to yield results that are too optimistic. ${ }^{17}$

The UTI care provided by Dutch GPs to male patients is as good as the care predicted by the diagnostic algorithm, which was based on dipstick test results and the patient's age. Empirical antimicrobial treatment can be initiated in the case of a positive nitrite test or when males above the age of 60 years consult with dysuria and fever $\left(>38^{\circ} \mathrm{C}\right)$. For all other males in whom UTI is suspected, GPs should await the urine culture result before starting antimicrobial treatment.

\section{Funding}

This work was supported by the Dutch Foundation of the Working Party on Antibiotic Policy (SWAB). The Dutch Sentinel GP network is supported by a grant from the Ministry of Health, Welfare and Sports (the Netherlands).

\section{Ethics committee}

Ethical approval was not required.

\section{Acknowledgements}

We thank all participating GPs and their patients. Without their help this study could not have been performed. We also thank Jacqueline Maes, Christel Driessen, Mayk Lucchesi, Jacqueline Peeters and Bram Vanderzanden for their excellent laboratory support.

\section{Provenance}

Freely submitted; externally peer reviewed.

\section{Competing interests}

The authors have declared no competing interests.

\section{Discuss this article}

Contribute and read comments about this article on the Discussion Forum: http://www.rcgp.org.uk/bjgp-discuss 


\section{REFERENCES}

1. Donker GA. Continuous morbidity registration dentinel general practice network. Annual report 2010. Utrecht: NIVEL, 2010. http://wnw.nivel.n//peilstations laccessed 19 Sep 2012).

2. Giesen LG, Cousins G, Dimitrov BD, et al. Predicting acute uncomplicated urinary tract infection in women: a systematic review of the diagnostic accuracy of symptoms and signs. BMC Fam Pract 2010; 11: 78.

3. Schmiemann G, Kniehl E, Gebhardt K, et al. The diagnosis of urinary tract infection: a systematic review. Dtsch Arztebl Int 2010; 107(21): 361-367.

4. Komaroff AL, Pass TM, McCue JD, et al. Management strategies for urinary and vaginal infections. Arch Intern Med 1978; 138(7): 1069-1073.

5. Mclsaac WJ, Low DE, Biringer A, et al. The impact of empirical management of acute cystitis on unnecessary antibiotic use. Arch Intern Med 2002; 162(5): 600-605.

6. Koeijers JJ, Kessels AG, Nys S, et al. Evaluation of the nitrite and leukocyte esterase activity tests for the diagnosis of acute symptomatic urinary tract infection in men. Clin Infect Dis 2007; 45(7): 894-896.

7. Hummers-Pradier E, Ohse AM, Koch M, et al. Urinary tract infection in men. Int J Clin Pharmacol Ther 2004; 42(7): 360-366.

8. van Pinxteren B, van Vliet SM, Wiersma TJ, et al. [Summary of the practice guideline 'Urinary-tract infections' (second revision) from the Dutch College of General Practitioners]. Ned Tijdschr Geneeskd 2006; 150(13): 718-722.

9. Winkens R, Nelissen-Arets H, Stobberingh E. Validity of the urine dipslide under daily practice conditions. Fam Pract 2003; 20(4): 410-412.
10. Lipsky BA, Ireton RC, Fihn SD, et al. Diagnosis of bacteriuria in men: specimen collection and culture interpretation. J Infect Dis 1987; 155(5): 847-854.

11. Isenberg HD. Essential procedures for clinical microbiology. Washington, DC: ASM Press, 1998.

12 Little P, Turner S, Rumsby K, et al. Developing clinical rules to predict urinary tract infection in primary care settings: sensitivity and specificity of near patien tests (dipsticks) and clinical scores. Br J Gen Pract 2006; 56(529): 606-612.

13. Eng J, Russell H. ROC analysis: web-based calculator for ROC curves Baltimore: Johns Hopkins University http://www.jrocfit.org laccessed 19 Sep 2012).

14. Ditchburn RK, Ditchburn JS. A study of microscopical and chemical tests for the rapid diagnosis of urinary tract infections in general practice. Br J Gen Pract 1990; 40(339): 406-408

15. Mclsaac WJ, Moineddin R, Ross S. Validation of a decision aid to assist physicians in reducing unnecessary antibiotic drug use for acute cystitis. Arch Intern Med 2007; 167(20): 2201-2206.

16. Caljouw MA, den Elzen WP, Cools HJ, et al. Predictive factors of urinary tract infections among the oldest old in the general population. A population-based prospective follow-up study. BMC Med 2011; 9: 57

17. Little P, Turner S, Rumsby K, et al. Validating the prediction of lower urinary tract infection in primary care: sensitivity and specificity of urinary dipsticks and clinical scores in women. Br J Gen Pract 2010; 60(576): 495-500. 